\title{
Speed Breeding in Agriculture Future Prospects
}

\author{
Ch. Sai Nayan Raju* and C. Kalyan Sagar \\ Department of Genetics and Plant Breeding, SHUATS Prayagraj, \\ UttarPradesh-211007, India \\ *Corresponding author
}

\section{A B S T R A C T}

\section{Keywords}

Speed breeding, Photoperiodism, Circadian-clock genes

\section{Article Info}

Accepted:

10 November 2020

Available Online:

10 December 2020
It is review highlighting the speed breeding future prospects Speed breeding is revolutionary tool of breeding technology by reducing crop generation time. It's a concept of extending the duration of plants daily exposure to light combined with early seed harvest to quickly from seed to seed by reducing the times of generation for some long day and day neutral crops. Basically, this experimental research was conducted in three methods they are Speed breeding I - Controlledenvironment chamber speed breeding. Speed breeding II - Glasshouse speed breeding conditions Speed breeding III - Homemade growth room design for low cost speed breeding. Developed by teams at the John Innes Centre, University of Queensland and University of Sydney. Dr Lee Hickey from the University of Queensland and Speed breeding concept was inspired by NASA's efforts to grow food on space stations which allows faster selection and population development. It was first described and implemented for wheat and peanut (Arachis hypogaea). Variations of this approach have been demonstrated to be an efficient system for rapid screening of wheat germplasm for adult plant resistance to various diseases and also for pyramiding multiple disease resistance in barley. This approach has also been adapted for high density plant production systems for SSD programs and also be created some of these artificial stimulating conditions used in this speed breeding technique the breeding technique uses modified temperature conditions and replacing the traditional artificial light like sodium vapour lamps or halogen lamps by LEDS light emitting diodes as a supplementary light source for extending photo periodism is used and to speed up the crossing and inbreeding of varieties speed breeding protocols designed different for different species altering the light treatments and modifying temperatures need to be optimized subsequently the optimal conditions to be followed in speed breeding in light spectrum that used PAR region of $(400-700 \mathrm{~nm})$ with mainstream focus on the blue red or far red regions of the spectra. A light/dark period was chosen over a continuous photoperiod to support functional expression of circadian clock genes. Specific photoperiod length of 22-h with 2-h of darkness is recommended to improve the plant health (for other species like wheat, barley, oat $18 \mathrm{~h}$ photo period is enough). Optimum temperature regime should be applied its aids in stress recovery. In most of the conditions at UQ, a $12-\mathrm{h} 22{ }^{\circ} \mathrm{C} / 17$ ${ }^{\circ} \mathrm{C}$ temperature cycling regime with $2 \mathrm{~h}$ of darkness occurring within $12 \mathrm{~h}$ of $17{ }^{\circ} \mathrm{C}$ has proven successful. Humidity, but a reasonable range of $60-70 \%$ is ideal. For crops that are more adapted to drier conditions, a lower humidity level may be advisable. A evaluation of speed breeding approaches was compared in two specific conditions of light like 22-h \& temperature 17 degree in glass house with some genotypes of wheat, barley, oat, Brachypodium distachyon (purple false brome)as a correspondence with other crops grown in glass house the crops which undergone the speed breeding with specific conditions has grown with other half time from other crops grown in normal glass house conditions. This plays pivotal role in plant breeding technology by meeting the needs of ever-growing population the speed breeding had some disadvantages over dwarf plants. The continuous exposure of light may deplete the trait of dwarfness in plants this should be the concerning trait of improvement for some major crops like one of them is rice (Oryza sativa). 


\section{Introduction}

Agriculture has always been responsible for producing food for the world population. It dates back well over 10,000 years ago, to the time of the hunter gatherer societies in Southwest Asia. Back then before the dawn of agriculture, it supported only4 million people. Today modern Agriculture now feeds over 7 billion people world-wide, so this statistic alone shows just how far the Industry has come in recent years (Tillman et al., 2002). The 2010 UN projections envisage global population to rise from 6.9 billion in 2010 to 8.1, 9.3 and 10.6 billion by mid-century under the low, medium or high assumptions (UN Population Division, 2011). The growing population in world is at alarming stage analysing present situations in the world we need to understand that population is increasing in arithmetic progression and to feed the dynamic population we need to adapt and innovate new methods of improving crop production with rapid pace in the similar fashion. To provide adequate food subsistence to the people living with diverse diet will require at least 0.5 hectare of arable land per person (Lal and Steward, 1990), and at this time, we have only 0.27 hectare per capita land available to us, which will drastically be reduced to 0.14 hectare per person within the next 40 years due to loss of land caused by population pressure (Pimentel, 1993; Pimentel et al., 1994; Pimentel et al., 1995; Pimentel, 1997). In his book titled 'World Soil Erosion and Conservation' published in the year 1993, David Pimentel mentioned that per capita shortage in the availability of land has remained the major reason for severe food shortage and malnutrition in many parts of the world. but in the available land we need to increase our production with various molecular approaches and with various other technologies and also land is stable factor which cannot be increased or decreased so the cultivation of crops per acre should be increased but because of many environmental factors like abiotic stress and biotic stress are influencing the crop yield ultimately reducing the crop productivity if you understand the statistics of food production in the world the production of food is immensely increased because of many agriculture universities or international institutes and their policies all around the globe but as of $21^{\text {st }}$ century the major concerning fact is that urbanization, climate change may pose possible threat to future of food security and the changing weather patterns over the years raises a serious concern about food security. it sometimes impossible to predict the consequences of climate change as result of climate change many institutes are starting climate resilient crop cultivation programs to adapt the dynamic climate parameters by developing various genomic technologies by various alternating technologies to conventional

Plant breeding and a because conventional breeding technology is very time consuming process to maintain the pace with growing population the crop production technology is to be enhanced or it should be more innovating way to speed up the crop life cycle by innovative breeding technology by reducing their generation time by creating artificial conditions called speed breeding, this technology is the advancement of conventional breeding technology. This concept was inspired from NASA efforts to grow crops in space in extra-terrestrial environment is fast tracking improvements in the range of crops using an enclosed chamber and extended photoperiod this opportunity was recognized and adapted to develop more rapidly to produce wheat and barley plants and allow faster selection and population development. later on speed breeding became norm in university of Queensland. Australia's' peed breeding allowing researchers to rapidly mobilise the genetic 
variation found in wild relatives of crops introduce in to elite varieties this original approach was first described for in two crops called wheat and peanut this approach have been demonstrated to be an efficient system for rapid screening of wheat germ plasm for adult plant resistance to various diseases and also for pyramiding multiple multiple disease resistance in barley.as of the research published in nature protocols This approach has also been adopted for high density plant production system for SSD programs. This "speed breeding" (SB) protocols has the potential to significantly accelerate breeding programs for different crops by reducing the generation time (Watson et al., 2018; VossFels et al., 2018). In SB, plants are grown in controlled environments with continuous light for $22 \mathrm{~h}$ per day at optimal temperature. The advantage of SB has been proven for many crops such as Brassica species, bread wheat, durum wheat, barley, chickpea, pea, grass pea, quinoa, oat, Brachypodium distachyon, and peanut; and at least four generations have been achieved in a single year using SB (O'Connor et al., 2013; Ghosh et al., 2018; Watson et al., 2018) and this particular technology uses some artificial conditions to grow a crop under modified temperature, humidity, photoperiod time this should or can be done in glass house conditions. the speed breeding technology along with these artificial conditions reduces the crop generation time much less if you compare the crop with normal glass house conditions the crop with these certain type of modifications can produces better results in that special glass house conditions the crop matures with in half time of crop grown in normal glass house conditions. Scientists at the john inn centre Earlham institute, and the quadram institute in Norwich UK, and the university of Queensland have improved this technique known as speed breeding\& adapting it to work in vast glass houses and in the scaled down desktop growth chambers This glass house experiment was conducted in (Norwich Uk) 2016 with No supplementary light or heat source given to the crop in early summer or spring. This experiment was conducted firstly in two main crops maize, wheat, these are the most important crops. Around the world maize stands first in the world with highest production around 1134 million thousand tones around the world maize stood first position among cereals and wheat stands second in the world production among cereals, pulses in many crops now this technology is widely exploited by many agricultural firms or research agencies by spending billions of dollars in many important crops like rice, pigeon pea, grass pea, chickpea and many more important crops.
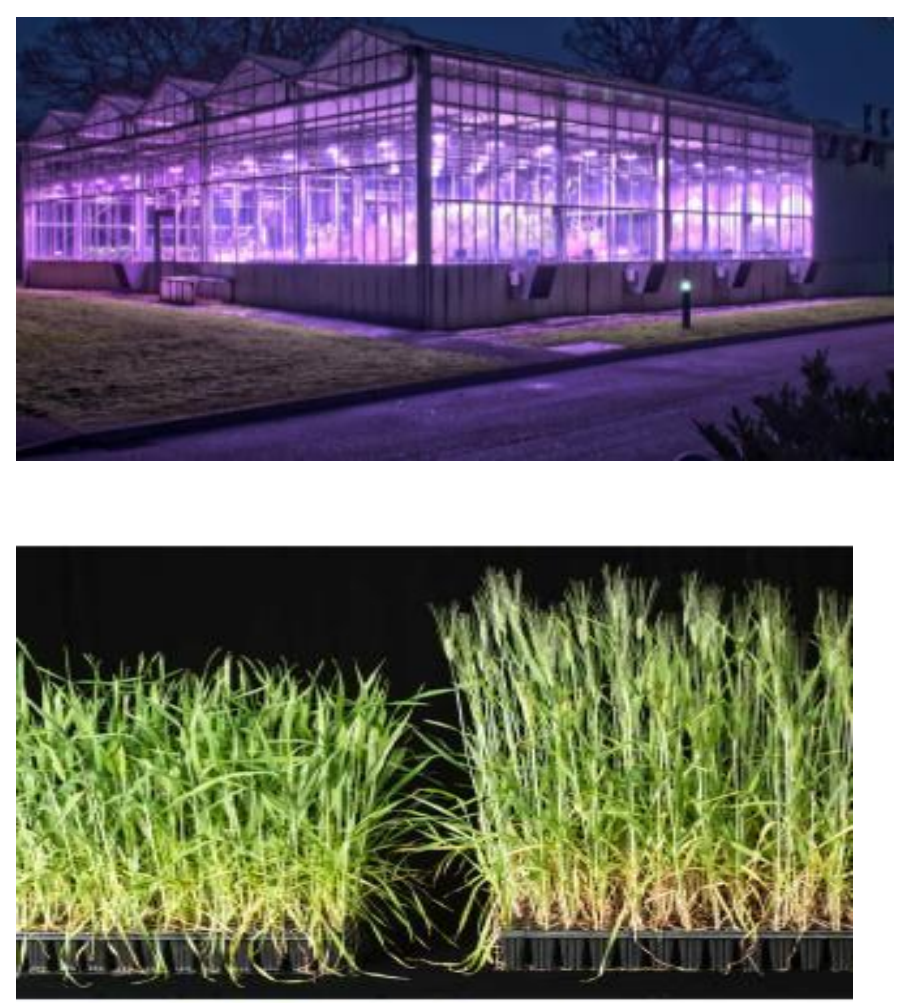

Ghosh et al., 2018

\section{Materials and Methods}

Speed breeding, I - Controlled-environment chamber speed breeding.

Speed breeding II - Glasshouse speed breeding conditions 
Speed breeding III - Homemade growth room design for low cost speed breeding.

\section{Speed breeding - I}

A conviron BDW chamber

Temperature: $22{ }^{\circ} \mathrm{C}$ during photoperiod of $22 \mathrm{hrs}$ and $17{ }^{\circ} \mathrm{C}$ during 2 hours of dark period

Humidity: $70 \%$

Lighting: Mixture of white LED bars, far-red LED lamps and ceramic metal hydrargyrum quartz iodine lamps

Light intensity was adjusted to $360-380$ umol $\mathrm{m}^{-2} \mathrm{~s}^{-1}$
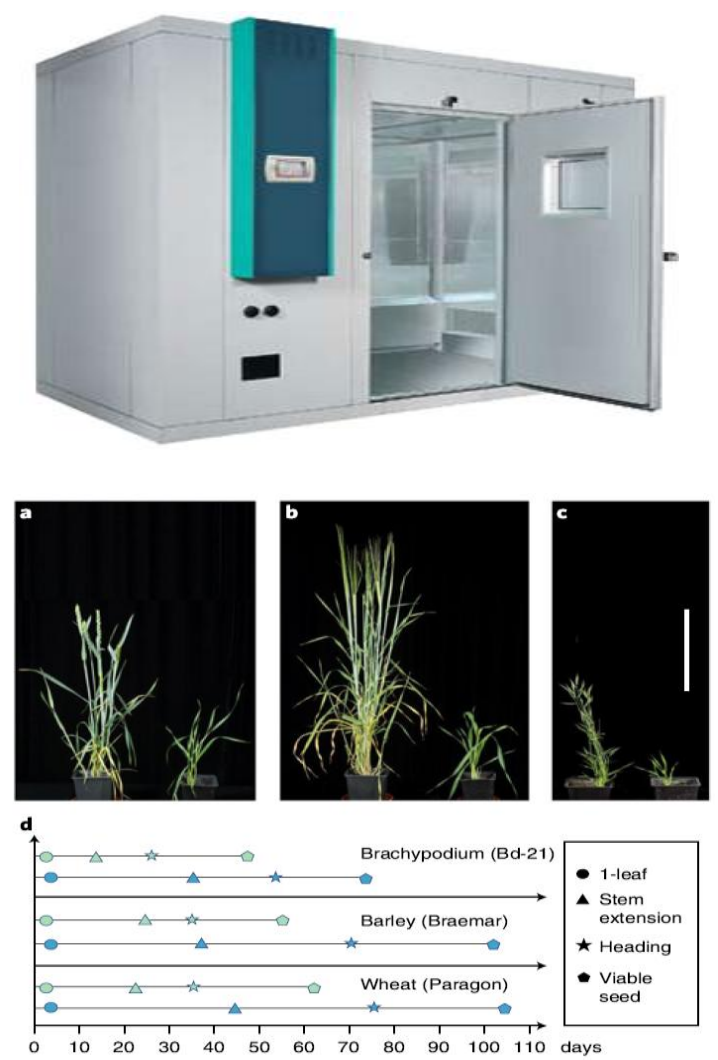

\section{Outcomes of speed breeding 1}

Anthesis in approximately half the time than those in green house

Seed count per spike decreased but not significantly

Plants produced healthy number of spikes per plant, despite of rapid growth
Viability of harvested seeds unaffected by speed breeding

\section{Speed breeding 2}

\section{Glasshouse speed breeding conditions}

A temperature-controlled glasshouse fitted with high pressure sodium vapour lamps (22 hours photoperiod)

Temperature: $17 / 22{ }^{\circ} \mathrm{C}$ regime with 12 hours turnover

Light intensity was set to $440-650 \mathrm{umol} \mathrm{m} \mathrm{m}^{-2} \mathrm{~s}^{-1}$ The two hours period without lamps operating and $17{ }^{\circ} \mathrm{C}$ cycle occurred during night
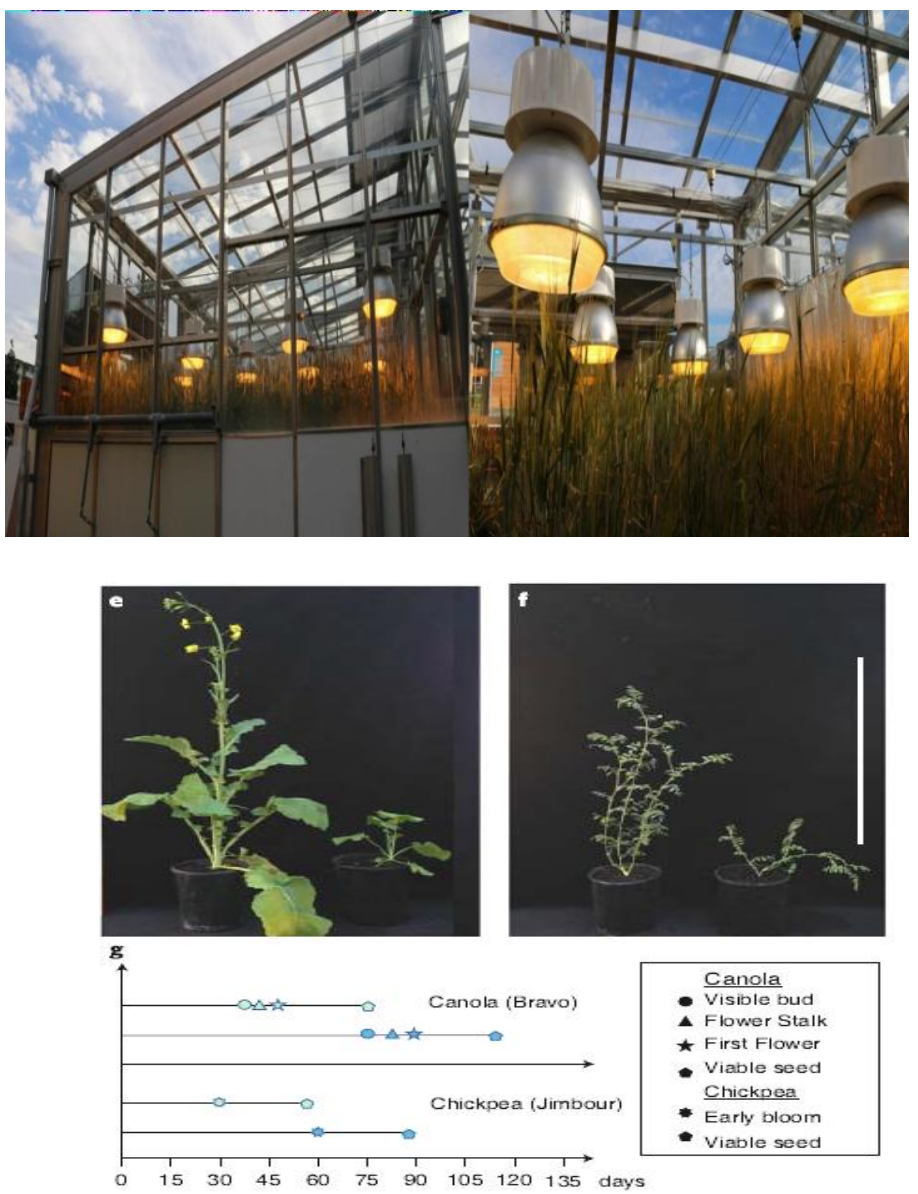

\section{Outcomes of speed breeding-2}

Wheat plants produced significantly more spikes than in day neutral condition. 
Grain number was unaffected by the rapid development in wheat and barley

Seed viability was either unaffected or improved compared to day neutral conditions

Seed production (g per plant) of canola and chickpea was similar.

\section{Speed breeding 3}

\section{Homemade growth room design for low cost speed breeding}

A room of $3 \mathrm{~m} \times 3 \mathrm{~m} \times 3 \mathrm{~m}$ with insulated sandwich panelling fitted with seven LB-8 LED light boxes

Lightning was set to run a 12- hours photoperiod for 4 weeks and then increased to an 18 hours

1.5 horsepower inverter split system domestic air conditioner $\left(18{ }^{\circ} \mathrm{C}\right.$ in darkness and $21{ }^{\circ} \mathrm{C}$ when LED lights on)

Automatic watering was achieved with irrigation controller

The cabinet has an available area of 0.225 $\mathrm{m} 2$. with the light levels in PPFD being on an average of $\sim 120 \mu \mathrm{mol} / \mathrm{m} 2 / \mathrm{s}$ at $16 \mathrm{~cm}$ above the base where the pots are kept, and $\sim 320$ $\mu \mathrm{mol} / \mathrm{m} 2 / \mathrm{s}$ and $220 \mu \mathrm{mol} / \mathrm{m} 2 / \mathrm{s}$ from a $10-\mathrm{cm}$ and $20-\mathrm{cm}$ distance, respectively, from the top of the cabinet where the lights are situated.

\section{Role of light on plants}

The light plays very pivotal role in the plants by effecting their photosynthetic process, flowering time and there is particular process in plants with respective to growth and developments of plants is called Photomorphogenesis.

Photoperiodism is the ability to use light to track time. Plants can tell the time of day and time of year by sensing and using various wavelengths of sunlight.

The response of plants to light is mediated by different photoreceptors, which are comprised of a protein covalently bonded to a lightabsorbing pigment called a chromophore. Together, the two are called a chromoprotein. The particular spectrum of visible light called red/far-red and violet-blue regions trigger structural development in plants. Sensory photoreceptors absorb light in these particular regions of the visible light spectrum because of the quality of light available in the daylight spectrum in terrestrial habitats, light absorption by chlorophylls peaks in the blue and red regions of the spectrum. As light filters through the canopy and the blue and red wavelengths are absorbed, the spectrum shifts to the far-red end, shifting the plant community to those plants better adapted to respond to far-red light. Blue-light receptors allow plants to gauge the direction and abundance of sunlight, which is rich in bluegreen emissions.

\section{The role of phytochrome in plants andthe Red/Far-Red Response}

The important phytochrome responses is regulated by the phytochrome system include photoperiodic induction of flowering, chloroplast development (not all synthesis of chloroplast occurs) leaf senescence and leaf abscission. The phytochromes are photoreceptors found in plants, bacteria, and fungi these belong to the family of chromoproteins with a linear tetrapyrrole chromophore, as also occurs for the chlorophylls and phycobilin's pyrrole refers to a five membered ring having 4 carbons, onenitrogen, and two double bonds phytochrome is an important pigment that regulates photomorphogenic aspects of plant growth and development such as seed germination, stem elongation, leaf expansion, formation of certain pigments, chloroplast 
development, and flowering. many of these processes can be saturated by low amounts of light, such, as $500 \mu \mathrm{molm}-2$ of red light(photons in the visible of 14 second of full sunlight). high irradiance level can also lead to effects mediated by phytochrome

\section{Phytochrome allows plant to sense the colour of light}

\section{Sunlight has a R:FR ratio of 1.2}

Light under a canopy of leaves has a R:FR ratio of 0.13

Light under $5 \mathrm{~mm}$ of soil has a R:FR ratio of 0.88

A higher proportion of FR lights allows plants detect when they are shaded

The structure of the linear tetrapyrrole is shown here phytochrome protein through sulphur linkage
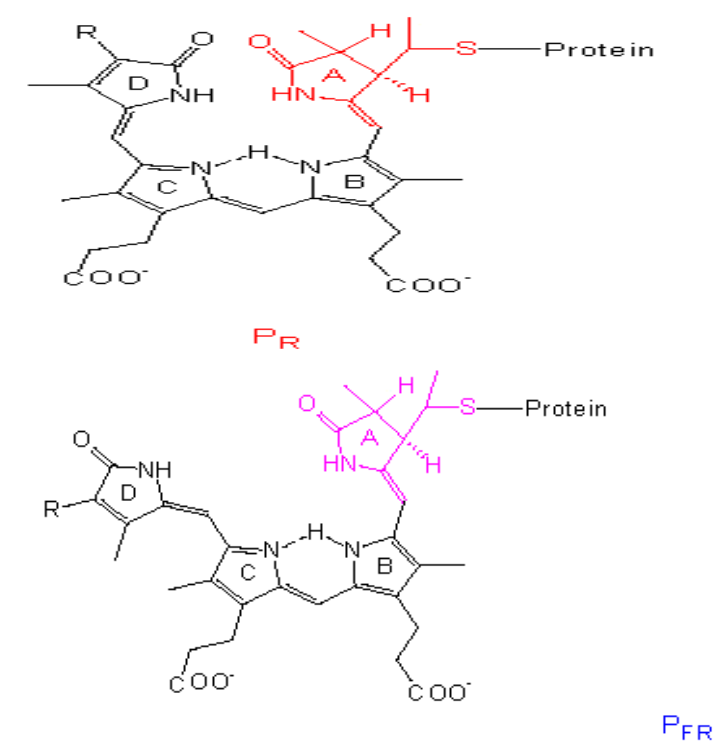

when plants are grown in full sunlight their stem elongation is more compared to when they are shadowed there are different phytochrome genes and proteins there are 5 phytochrome genes in dicots in Aradopsis genes termed phyA, phyB, phyC, phyD, phyE PhyB is helps for daylength detection in flowering

Light absorption leads to a photoisomerization about the double bond between rings $\mathrm{C}$ and $\mathrm{D}$, which apparently underlies the conversion of phytochrometoits physiologically active form (Fig. 1-9). Photoisomerization: is the photochemical cistrans isomerization of organic molecules with a double bond in their structure. it's a common photoreaction.
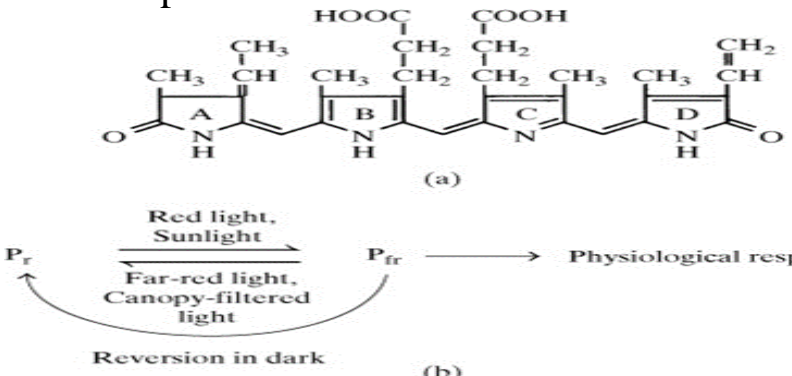

Phytochrome structure, interconversions, and associated physiological responses. (a) Structure for $\mathrm{P}_{\mathrm{r}}$, indicating the tetrapyrrole forming the chromophore and the convention for lettering the rings; and (b) light and dark interconversions of phytochrome, indicating reactions promoted by the physiologically active form, $\mathrm{P}_{\mathrm{fr}}$, such as promotion of seed germination, inhibition of etiolation (excess stem elongation in low light), promotion of leaf expansion, and inhibition of flowering.

Basically, Phytochromes have two photointerconvertible forms: $\mathrm{Pr}$ and Pfr. $\mathrm{Pr}$ it is form synthesised in dark brown seedlings absorbs red light $(\sim 667 \mathrm{~nm})$ and itis immediately converted to Pfr. Pfr is an active form initiates biological responses when Pfr absorbs red light it is converted to pr form basically pfr absorbs far-red light $(\sim 730 \mathrm{~nm})$ and is quickly converted back to Pr.pfr is spontaneously revert to the pr form in the dark over time is called dark reversion pfr is susceptible to proteinases pfr absorbs some red light there is balance of $85 \% \mathrm{pfr}$ and $15 \%$ pr. pr absorbs very little far red light so in 
far red-light there is balance of $97 \%$ red-light and $3 \%$ pfr

Absorption of red or far-red light causes a massive change to the shape of the chromophore, altering the conformation and activity of the phytochrome protein to which it is bound. Pfr is the physiologically active form of the protein; therefore, exposure to red light yields physiological activity. Exposure to far-red light inhibits phytochrome activity. Together, the two forms represent the phytochrome system (Figure 1).

The phytochrome system acts as a biological light switch. It monitors the level, intensity, duration, and color of environmental light. The effect of red light is reversible by immediately shining far-red light on the sample, which converts the chromoprotein to the inactive Pr form. Additionally, Pfr can slowly revert to Pr in the dark, or break down over time. In all instances, the physiological response induced by red light is reversed. The active form of phytochrome (Pfr) can directly activate other molecules in the cytoplasm, or it can be trafficked to the nucleus, where it directly activates or represses specific gene expression.

\section{$P_{R} \quad P_{F R} \quad$ biological activity}

Once the phytochrome system evolved, plants adapted it to serve a variety of needs. Unfiltered, full sunlight contains much more red light than far-red light. Because chlorophyll absorbs strongly in the red region of the visible spectrum, but not in the far-red region, any plant in the shade of another plant on the forest floor will be exposed to reddepleted, far-red-enriched light. The preponderance of far-red light converts phytochrome in the shaded leaves to the Pr (inactive) form, slowing growth. The nearest non-shaded (or even less-shaded) areas on the forest floor have more red light; leaves exposed to these areas sense the red light, which activates the Pfr form and induces growth. In short, plant shoots use the phytochrome system to grow away from shade and towards light. Because competition for light is so fierce in a dense plant community, the evolutionary advantages of the phytochrome system are obvious.

Plants also use the phytochrome system to sense the change of season. Photoperiodism is a biological response to the timing and duration of day and night. It controls flowering, setting of winter buds, and vegetative growth. Detection of seasonal changes is crucial to plant survival. Although temperature and light intensity influence plant growth, they are not reliable indicators of season because they may vary from one year to the next. Day length is a better indicator of the time of year.

As stated above, unfiltered sunlight is rich in red light but deficient in far-red light. Therefore, at dawn, all the phytochrome molecules in a leaf quickly convert to the active Pfr form, and remain in that form until sunset. In the dark, the Pfr form takes hours to slowly revert back to the Pr form. If the night is long (as in winter), all of the Pfr form reverts. If the night is short (as in summer), a considerable amount of $\mathrm{Pfr}$ may remain at sunrise. By sensing the Pr/Pfr ratio at dawn, a plant can determine the length of the day/night cycle. In addition, leaves retain that information for several days, allowing a comparison between the length of the previous night and the preceding several nights. Shorter nights indicate springtime to the plant; when the nights become longer, autumn is approaching. This information, along with sensing temperature and water availability, allows plants to determine the time of the year and adjust their physiology accordingly. Short-day (long-night) plants use 
this information to flower in the late summer and early fall, when nights exceed a critical length (often eight or fewer hours). Long-day (short-night) plants flower during the spring, when darkness is less than a critical length (often eight to 15 hours). Not all plants use the phytochrome system in this way. Flowering in day-neutral plants is not regulated by daylength.

Importance \& role of supplimentry light system with modtification of red and far red light used in speed breeding technology

The plants which are subjected to speed breeding technology comes to flowering less than half time compared to the crops which are grown in normal greenhouse conditions. In this technology particularly $22 \mathrm{hr}$ photoperiod is achieved by using LED illumination as supplementary source of light. They used a patented spectra called Volya's NS1 it is close replica of closer wavelength of produced by sunlight on clear sky day additionally they used far red spectrum with NS1 so as to induce flowering. The low red; far red ratio has shown reduced time to flowering in plant species in wheat, barley, grain legumes. Photoperiod of $22 \mathrm{~h}$ followed with $2 \mathrm{~h}$ of darkness in $24 \mathrm{~h}$ diurnal cycle but according to experiment dark period slightly improves the plant health but $18 \mathrm{~h}$ photoperiod was sufficient to achieve faster generation times for wheat, barley oat and triticale Other lightening sources can be used like halogen lamps or in the case of glass house by supplementing with ambient light with LEDS OR SVLS

In addition to control the lighting quality they recommended PPFD of $450-500 \mu \mathrm{mol} / \mathrm{m} 2 / \mathrm{s}$ at [plant canopy height suggested amount of light is actually affective, crops species vary in their response to high irradiance the modification of ratio is had drastic effect on crop with the lowering the ratio has accelerated the flowering process in plant the measurements of light spectrum should be taken before the commencement of the speed breeding experiment the photosynthetically active radiation PAR region(400-700nm) with a particular focus on blue and red and far red ranges is suitable to use in speed breeding

The $22 \mathrm{~h}$ approach demonstrated to be suitable for accelerating research activities involving adult plant phenotyping, genetic structuring, molecular studies like gene transformation, [and] rapid generation advancement [in Wheat, barley]. It was further demonstrated to be suitable for rapid generation advancement for pea, B. distachyon, M. truncatula, canola and chickpea." (Ghosh, 2018).

Disadvantages of usage low r/fr:

From all the experiments conducted in experimental field faculty of agronomy university of Buenos Aires, Argentina conducted experiment on the responds to low red light /far red light stimulating dense stands were evaluated in wheat cultivars released in different times of $20^{\text {th }}$ century the tillering responses to low $\mathrm{R} / \mathrm{FR}$ reduced grain yield per plant (primarily grain number and secondarily grain weight per plant) particularly in modern cultivars. low R/FR ratios delayed the spike growth and development accelerated the development of florets already initiated and reduced the number of fertile florets at anthesis

\section{Role of temperature in plants}

For every crop there will be an optimal temperature regime (maximum and minimum temperature) temperature plays very crucial role in the plant growth

There are various effects of temperature on plant growth is

1) important for growth and development

2) optimum temperature is required for dry 
matter accumulation

3) high night temperature for -growth of shoot

For every crop there is maximum minimum and temperature called cardinal temperature points optimum temperature range is very important

\begin{tabular}{|l|l|l|}
\hline Wheat & $3-4^{\circ} \mathrm{c}$ & Minimum \\
\cline { 2 - 3 } & $25^{\circ} \mathrm{c}$ & optimum \\
\hline & $30^{\circ} \mathrm{c}-32^{\circ} \mathrm{c}$ & maximum \\
\hline
\end{tabular}

There are various serious consequences if the temperature deviated from optimal temperature of the crop

1) low temperature injuries like chilling injury, freezing injury, suffocation, heaving 2) high temperature injuries effects like mineral temperature, shoot growth, pollen development, scorching

Cardinal temperature values for selected annual (non-perennial) crops are given in Hatfield et al., (2008), Hatfield et al., (2011) for different species.

In general, extreme high temperatures during the reproductive stage will affect pollen viability, fertilization, and grain or fruit formation (Hatfield et al., 2008, Hatfield et al., 2011).

Given the negative impacts of high temperatures on pollen viability, recent observations from Shah et al., (2011)

For peanut (and potentially other legumes) the sensitivity to elevated temperature for a given flower, extends from 6 days prior to opening (pollen cell division and formation) up through the day of anthesis (Prasad et al., 2001).

Role of temperature in speed breeding

\section{technology}

The optimal temperature regime is also applied here in speed technology there is usage of higher temperature during prolong exposure to photoperiod whereas fall in temperature and some dark period helps stress recovery of plants from the stress caused by prolonged exposed to temperature at university of Queensland, Australia the photoperiod length is a $12-\mathrm{h} 22^{\circ} \mathrm{c} / 17{ }^{\circ} \mathrm{c}$ temperature cycling regime with 2 -h of darkness accruing within $12-\mathrm{h} 22^{\circ} \mathrm{c} / 17^{\circ}$ for 22-h of light and 2-h of darkness Chas proven successful other breeding's me.

\section{Need of speed breeding}

The need of speed breeding in current era Is very much essential because of various disadvantages in current plant breeding technologies the present breeding technology is much slower process to develop variety it take years to release a new variety and availability of variability among genotypes are depleting because of excessive selfpollination\& homozygosity in plants. The biggest challenge of breeding higher yielding and more resilient crops is the inability to complete more generations in lesser time Certain crop species, such as radish (Raphanus sativus), pepper (Capsicum annum), and leafy vegetables such as Amaranth (Amaranthus spp.) and sunflower (Helianthus annuus) responded positively to increased day length. Speed breeding of shortday crops has been limited because of their flowering requirements. It is possible to develop successive generations of improved crops for field examination via SSD, which is cheaper compared to the production of DHs. Plant-pathogen interactions, plant anatomy and flowering time can be studied in detail and repeated using the technology. For the photosensitive crops like soybean, speed breeding is not suitable to speed up the 
breeding cycles.to overcome that we need powerful breeding technology to increase the pace of breeding technologies as well as developing much quality and new cultivars which helps to cope up current changing climate

\section{Earlier approaches to hasten breeding} cycles

\section{Shuttle breeding}

The objective of speeding the process by growing two successive plantings per year

\section{Double haploids technology (DH)}

Generated directly from pollen or egg cells, subsequently chromosome doubling to restore fertility and the normal diploid chromosome number.

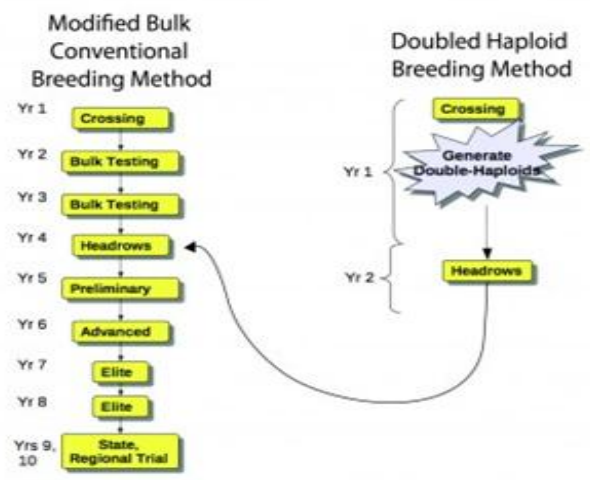

\section{Effect of speed breeding on SSD}

Wheat and barley genotypes were grown in 100-cell trays under both speed breeding and 12-hour day-neutral photoperiod conditions in the glasshouse.

The equated density of approximately 900 plants per $\mathrm{m}^{2}$

Generation time was shorter than for plants grown at lower density in previous speed breeding experiments

Higher density may cause stress or plant competition, lead to hasten flowering Integrating speed breeding and SSD techniques can effectively accelerate the generation of inbred lines for research and plant breeding programs.

\section{Effect of speed breeding on phenotypic ability}

The phenotypes associated with the ethyl methane sulfonate-induced mutation of the awn suppressor B1 locus9 and the Green Revolution Reduced height (Rht) genes in wheat 10 could be accurately recapitulated in the controlled environment room conditions.
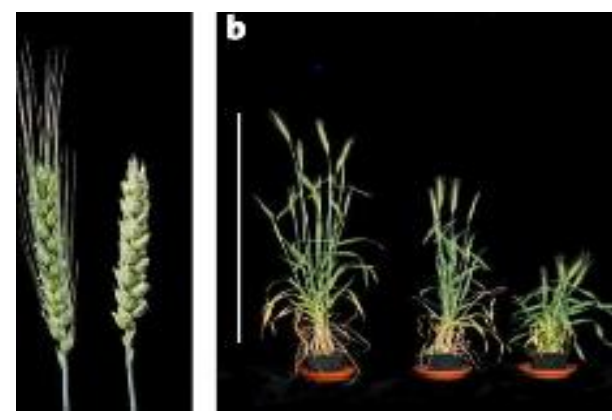

Evaluated the effect of loss of function of FLOWERING LOCUS T-B1 in the F6 recombinant inbred line of Paragon x W352 under speed breeding conditions. And observed the expected late flowering phenotype in the recombinant inbred line, albeit within fewer days and with fewer leaves produced.

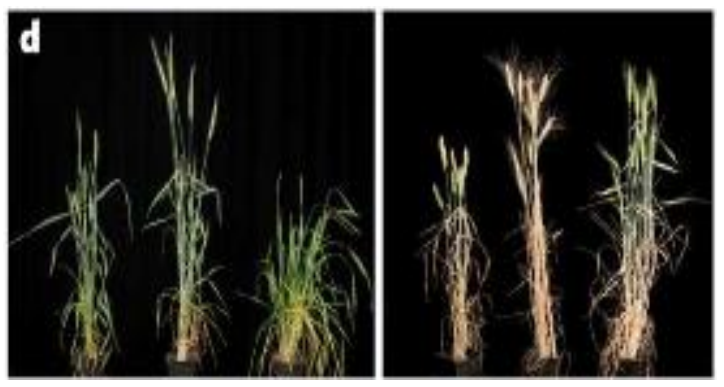

Wheat spikes with Fusarium graminearum, the causal agent of fusarium head blight (FHB) also found clear signs of FHB progression in the susceptible cultivar, and 
little to no disease progression in the resistant cultivar

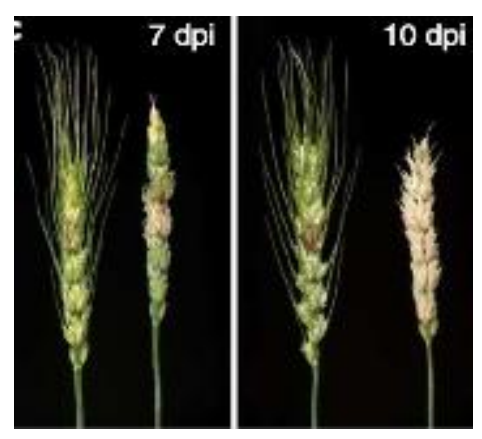

Effect of speed breeding on cuticular $\beta$ diketone wax production in barley. The mutant leaf sheaths exhibited a clear, decreased glaucous appearance in the flag.

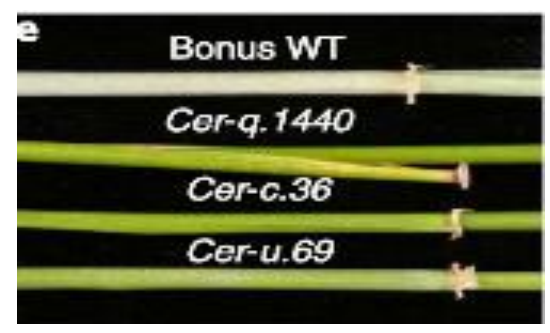

Speed breeding as a tool in other breeding methods

Speed breeding can be used as a rapid generation advancement tool in other conventional breeding methods to advance a generation in very less time in crops by using this technology in different phases of other breeding methods. conventional breeding technology along with speed breeding technology helps to maintain good relationship with nature and improves the speed of current conventional breeding technology in general in traditional breeding method of plant breeding we can yield of 1 or 2 maximum but by using speed breeding technology we can boost up the pace of traditional breeding methods for to completing 6 generations of crop within year and jump in to next crop this advance tool rapidly drives and improves and save time by developing a variety with pace along with growing population around the globeit's also helps to maintain the stability of crops and increase the productivity, food security rapidly

\section{Combining speed breeding technology with other state-art-technologies}

Speed breeding has a lot of potential to completely change present scenario of current plant breeding technologies by combining with state art technologies like with other molecular approaches, genome editing technologies like CRIPSPR-CAS9 its saves lot of time and it helps the plant breeders snip out the yield reducing or other vulnerable traits from the crop and advancing the crop generation with rapid pace speed breeding can be done in diploid crops then polyploid crops because of their complex genomics

Speed breeding can also be utilized to boost up the transgenic approaches of crop improvement.

Scientists are also applying speed breeding system to speed up the double haploid program.

The approach has also been adapted for high density plant production systems for SSD programs

Generation time was shorter than for plants grown at lower density in previous speed breeding experiments

Higher density may caused stress or plant competition, lead to hasten flowering

Speed breeding and marker assisted selection: For genetically well-defined traits, speed breeding could be used to rapidly introgress genes or haplotypes into elite lines using marker-assisted selection.

The speed breeding system is potentially relevant for the rapid development of RIL's which are essential for molecular marker discovery. 
Speed breeding and Association mapping: To track and confirm the presence of target regions.

Speed breeding and genomic selection: Pyramiding of multiple traits and to enable selection for yield and grain quality traits.

The speed breeding / SSD system is ideally suited to a backcrossing breeding strategy.

\section{Increasing genetic gain of the crop}

Modern breeding and advance management practices have contributed to the crop 0-2.-0.8 $\%$ annually in crop productivity but this is not sufficient for current growing population because of varied factors like soil, climate and environment weather patterns in particular regions or specific regions some crops of particular regions have attained yield plateaus i.e. such plateaus were evident in wheat and rice yields before the exploitation of semi dwarfing genes and being experienced in once again in some places in such cases we need to introduce new variability in breeding populations in order to break the plateau due to lack of genetic base and lack of potential to increase harvest index of elite breeding stocks of the crops sometimes due to harsh climatic factors may affect sustained yield factor in crops and sudden resurgence of pests and pathogens among crops due to hampered weather conditions but Increasing 2\% genetic gain of crop is huge challenge in essence the genetic gain crop of the determined by following equation

$\Delta G=i h \sigma \mathrm{A} / L$,

Whereas $i=$ selection intensity, $h$ is the square root of the heritability in the narrow sense $\sigma A$ is square root of additive genetic variance and $\mathrm{L}$ is the length of breeding cycle interval or generation and improvements in selection accuracy and intensity can lead to minor changes in genetic gain perspective the introduction of new favourable genes through rapid breeding cycles seems to possible senior to boost genetic gain crops could help to achieve the food challenge of the 2050

\section{The importance of rapid breeding cycles}

The climate change is happening rapidly best predictor is current changes in climate so we need to develop climate smart cultivars with rapid breeding cycles we need to replace old varieties with new cultivars the farmers working on cropping patterns governed by highly competitive seed industries by population improvement by selecting the crops of high phenotypic and genotypic value which are intermated to from a new breeding cycle which is superior or incrementally improved to previous cycle as result of changed allele frequencies it is the component of breeding system

Breeding system is based on quantitative principles significantly shorten breeding cycles by reducing excessive testing before selection, adoption of breeding tools such as rapid-generation advance in off-season nurseries or controlled environments, doubled-haploid technology, and genomic selection ((O'Connor et al., 2013; Prigge et al., 2011; Heffner et al., 2010) the most common breeding scheme in the world is pedigree system of breeding it almost take 12 years to complete without off seasons nurseries technology the breeding length can be reduced by $30 \%$ by adding off season nursery technology 
Fig.1 A set-up for speed breeding using LED lighting

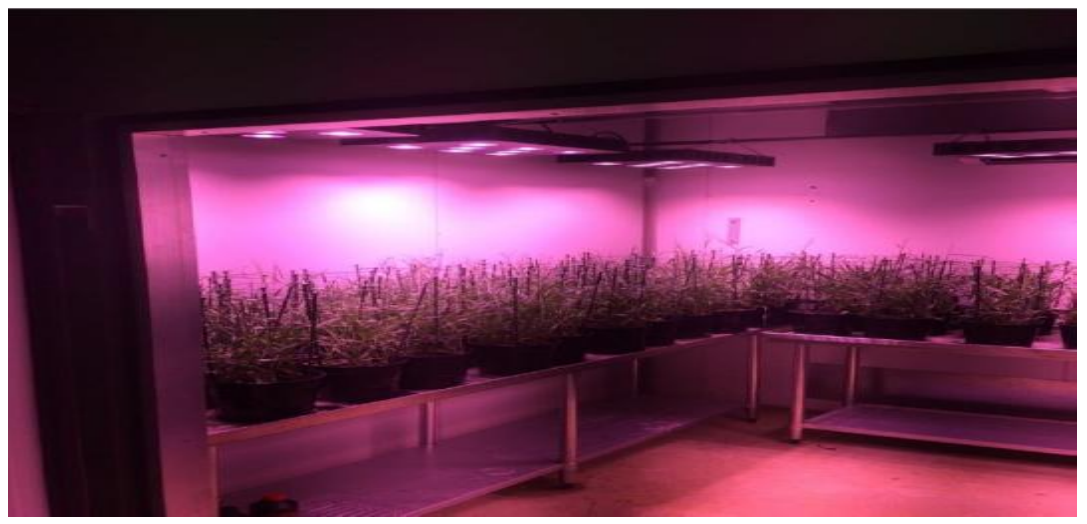

Fig.2 Bench top cabinet
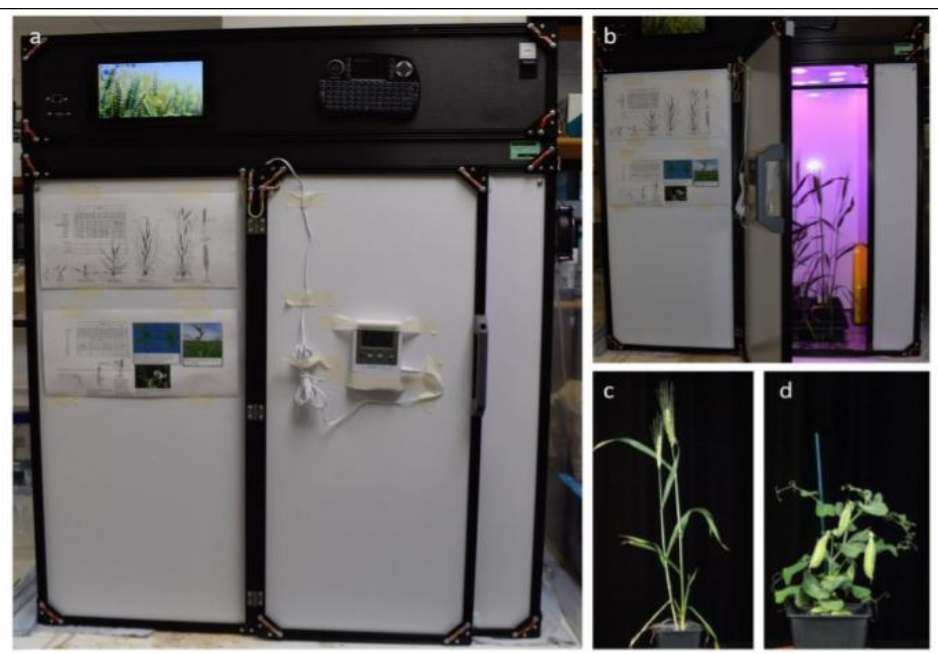

Fig.3 Layout of the glasshouse used for speed breeding at the John Innes Centre, UK
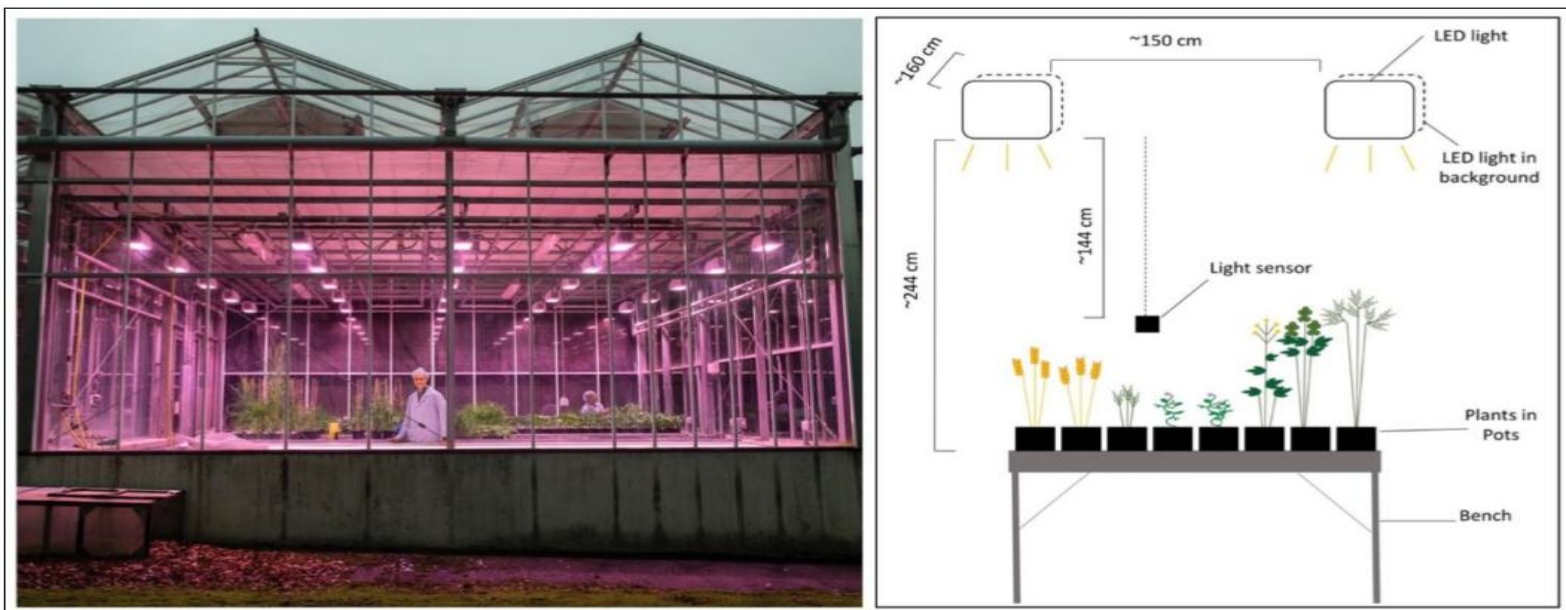
Fig.4 Layout of the glasshouse used for speed breeding at The University of Queensland, Australia Ghosh et al., 2018
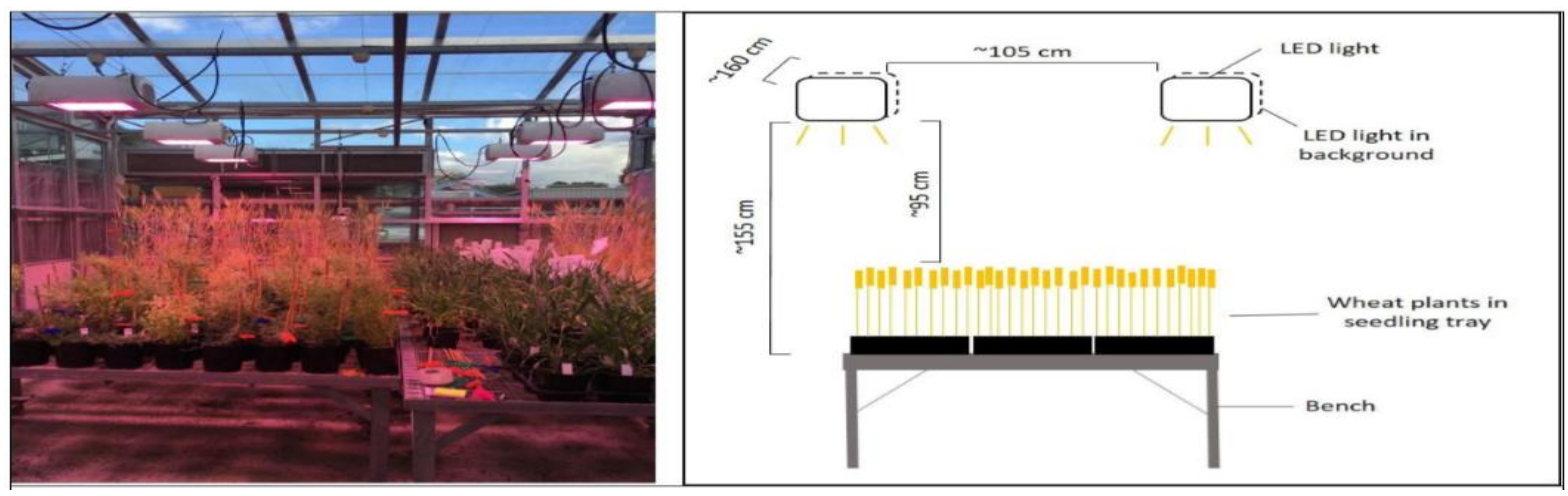

Fig.5 The biologically inactive form of phytochrome (Pr) is converted to the biologically active form Pfr under illumination with red light. Far-red light and darkness convert the molecule back to the inactive form.

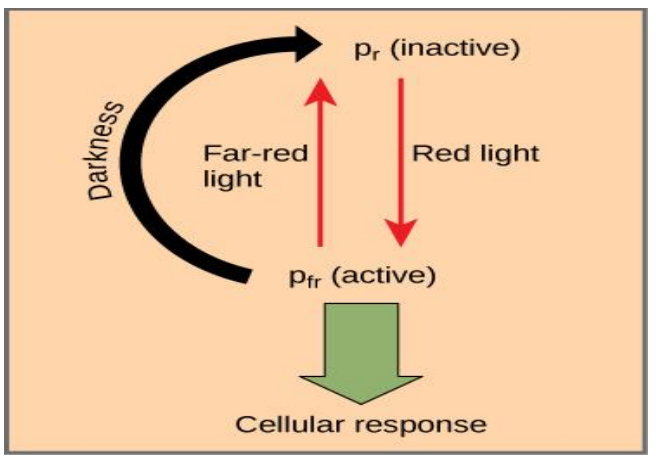

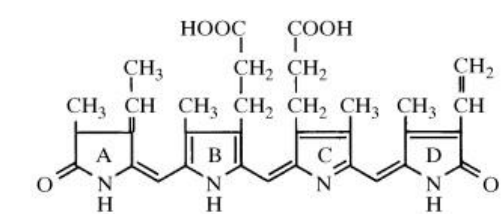

(a)

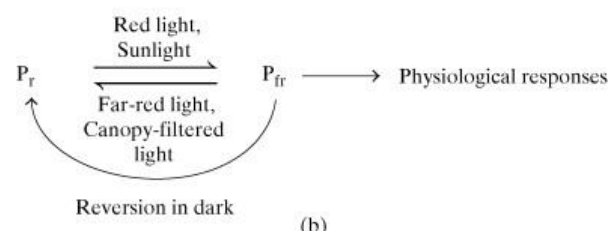

(b)

Fig.6 Current pipeline for development of verities

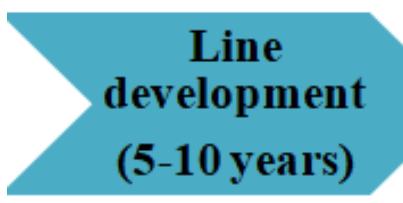

Field testing

\section{Release}

(3-5 years)

(1-3 years)

Speed breeding targets here

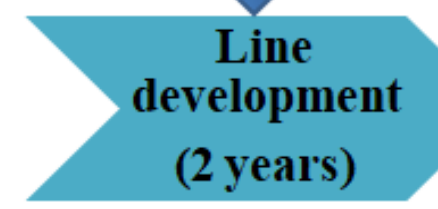

Field testing

\section{Release}

(3-5 years)

\section{(1-3 years)}


Fig.7

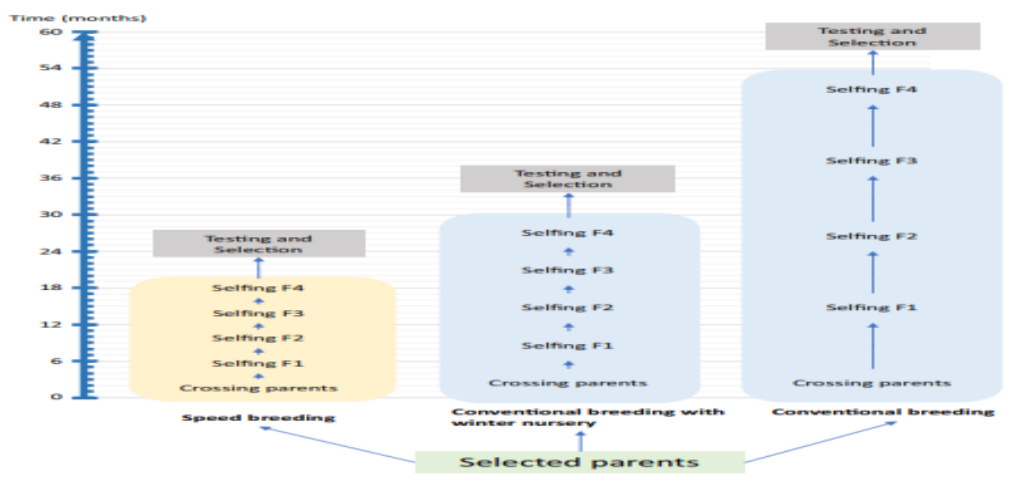

Chiurugwi et al., 2018

Fig.8 F2 plants growing in the greenhouse under controlled environment conditions at 15 days after sowing

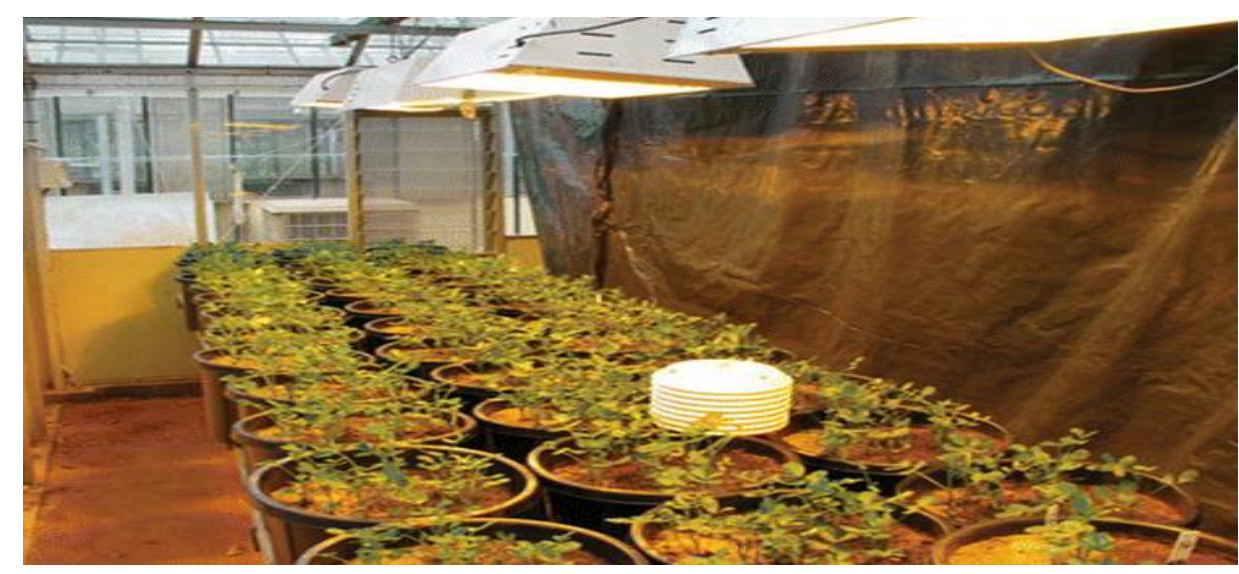

Fig.9

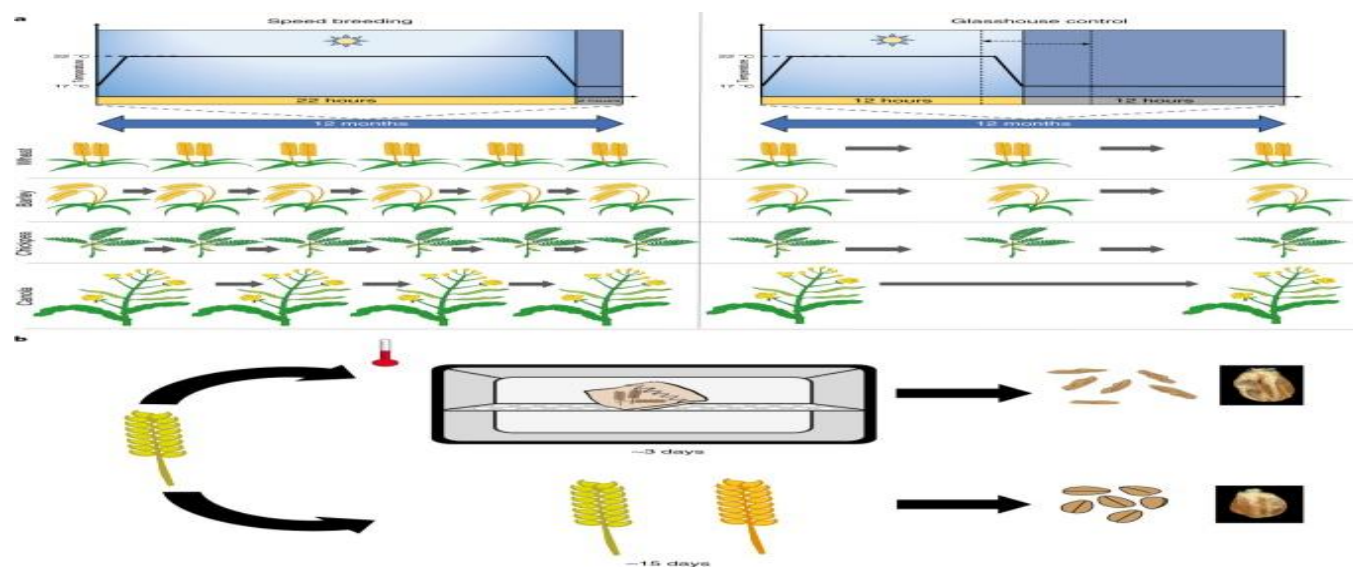




\section{Potential advantages of speed breeding}

Scientist can study this technology the way it deals with diseases and their shape and structure and flowering time and from the above we can know the growing cycle can be repeated every 8 weeks

The quality and yield of the plants can be grown under controlled climate and extended day light conditions sometimes better than those of crops which are grown under normal glass house conditions In future this technology can be used in various crops and it many other applications it can applied to vertical farming gardens, horticultural crops

From the result to know the being able to produce more generations in year will allow to test and create for new genetic combinations looking for best combinations of environments This technology will potentially allow us to study about flowering time and plant pathogen interactions shape and structure

\section{Limitations}

Short-day (SD) plants require the photoperiod to be less than the critical day length to flower which could be at odds with SB conditions. The SB procedures presented here take place in an enclosed, artificial environment, which differs significantly from the field

Early harvest of immature seed can interfere with the phenotyping of some seed traits.

The initial investment is high

\section{Core goals of speed breeding}

Why we actually need of this advance technology because of rapid growing population, climate change, rapid urbanization, depleting land are the major concerns to develop this technology if land is depleting we need to increase the food production per acre or ha and developing horticultural crops, and drought resistant crops and increase the genetic gain of the crop by adapting various technologies one such technology came in to existence is speed breeding this technology does need much need of land but it can advance the crop generation with rapid pace this technology will feed the future growing population

\section{Extending speed breeding technology to numerous crops}

Currently speed breeding technology limited to some crops like it was done in some of the important crops like wheat, barley chickpea and canola but we need to extend this powerful technology to various crops including horticultural crops and not only in reducing the life cycles of crops but also, we need to develop this technology in to fullfledged breeding method in to various crops

In conclusion the world population is relentlessly increasing, while there is consistent decline in the environment to which crops are exposed for example there is mounting water scarcity, decreasing area of cultivable land, increasing pollution and pollution and environmental degradation consistent emergence of new pathotypes and biotypes of pathogens and insect, pests, shortage of nutrients and possible adverse effects of climate change. These necessitate concerted efforts and innovative programmes of crop improvement and their chief focus should be stability and sustainability of crop yields. the traits important for yield stability and sustainability would include resistance /tolerance to abiotic and biotic stresses, and nutrient and water use efficiency, in addition, varieties suitable for cultivation on marginal lands will have to be developed and greater emphasis may have to be placed or improving minor crops (Collard and Mackill, 2001). 
The past achievements of plant breeding fully illustrate the future possibilities. modifications in the genetic makeup of crops, therefore may be expected to be quite rewarding in the future as well. there is some evidence suggesting that rate of increase in crop yields is declining: this emphasizes the need for novel tools. genetic engineering is gaining importance and we already have genetically engineered varieties in commercial cultivation. genes from varied organisms may be expected to boost the performance of crops especially with regard to their resistance to biotic and abiotic stresses. in addition, crop plants are likely to be cultivated for recovery of valuable compounds like pharmaceuticals produced by genes introduced into them through genetic engineering. it may be pointed out that in Europe hirudin an antithrombin protein is already being produced from transgenic Brassica napus. genetic engineering is expected to be increasingly used to develop varieties with novel features such varieties are likely to become widely adopted

\section{Future of speed breeding}

Speed breeding likely to reduce generation time for other crop species, such as sunflower, pepper and radish which have been shown to respond well to extended photoperiod.

Direct application of speed breeding protocols to short-day species such as maize or rice are unlikely to be successful.

Advance in LED light technologies, provides opportunity to optimize PAR and customize the wavelength and intensity to suit different growth stages and plant species.

\section{References}

Anthony Hall, László Kozma-Bognár, Réka Tóth, Ferenc Nagy, and Andrew J. Millar ${ }^{*}$ Conditional Circadian Regulation of Phytochrome A Gene
Expression

Cristina Cecilia Ugarte, Santiago Ariel Trupkin, Hernán Ghiglione, Gustavo Slafer and Jorge José Casal. Low red/far-red ratios delay spike and stem growth in wheat

D.J. O'Connor, G.C. Wright, M.J. Dieters, D.L. George, M.N. Hunter, J.R. Tatnell, D.B. Fleischfresser Development and Application of Speed Breeding Technologies in a Commercial Peanut Breeding Program Peanut Sci., 40 (2013), pp. 107-114, 10.3146/PS1212.1CrossRefView Record in Scop

Gerald F. Deitzer, Rebecca Hayes, and Merten Jabben Kinetics and Time Dependence of the Effect of Far Red Light on the Photoperiodic Induction of Flowering in Wintex Barley.

Ghosh, S., Watson, A., Gonzalez-Navarro, O. E., Ramirez-Gonzalez, R. H., Yanes, L., Mendoza-Suárez, M., et al., (2018). Speed breeding in growth chambers and glasshouses for crop breeding and model plant research. Nat. Protoc. 13 (12), 2944-2963. doi: 10.1038/s41596018-0072-Z

Ghosh, S., Watson, A., Gonzalez-Navarro, O.E. et al., 2018. Speed breeding in growth chambers and glasshouses for crop breeding and model plant research. Nat Protoc 13, 2944-2963 doi:10.1038/s41596-018-0072-z https://www.biorxiv.org/content/biorxiv /early/2018/07/16/369512.full.pdf

Global Climate Report - January 2020

Holland RW, Vince D. Floral initiation in Lolium temulentum L.: the role of phytochrome in the responses to red and far-red light.

Lal, R. (1989). Land Degradation and its Impact on Food and other Resources. In D. Piementel (Ed.), Food and Natural Resources. San Diego: Academic press.

Leyla Bayat, Mostafa Arab, Sassan Aliniaeifard, Mehdi Seif, Oksana 
Lastochkina, Tao LiEffects of growth under different light spectra on the subsequent high light tolerance in rose plants

Mary H. Dyer, Does Weather Affect Plant Growth: Effect of Temperature on Plants

MINI NI Integration of light signalling with photoperiodic flowering and circadian rhythm

O'Connor, D. J., Wright, G. C., Dieters, M. J., George, D. L., Hunter, M. N., Tatnell, J. R., et al., (2013). Development and application of speed breeding technologies in a commercial peanut breeding program. Peanut Sci. 40 (2), 107-114. doi: 10.3146/PS12-12.1

Piementel et al., (1994). Natural Resources and Optimum Human Population. Population and Environment, 15, 11171123. http://dx.doi.org/10.1126/science.267.5 201.1117

Prof Gert Venter Light and its effects on plant growth

Steffi Noack, Tilman Lampeter, in Methods in Enzymology, 2007 Two-Component Signalling Systems, Part B

Tillman, D., Cassman, K. G., Matson, P. A., 2002. Agricultural sustainability and im-proved production practices. insight review articles, 671-676

UN Population Division (2011a) World population prospects: the 2010 revision. New York Walpole SC, Prieto-Merino D, Edwards P et al., (2012) The weight of nations: an estimation of adult human biomass. BMC Public Health 12(1):439.

Valoya article on The Role of LEDs in Speed Breeding https://www.valoya.com/therole-of-leds-in-speed-breeding/ (LED supplier of the John Innes Centre)

Voss-Fels, K. P., Herzog, E., Dreisigacker, S., Sukumaran, S., Watson, A., Frisc, M., et al., (2018). "SpeedGS" to Accelerate Genetic Gain in Spring Wheat," in Applications of Genetic and Genomic Research in Cereals. Eds. Miedaner, T., Korzun, V. (United Kingdom: Woodhead Publishing), 303-323. doi: 10.1016/B978-0-08-102163-7.00014-4

Watson, A., Ghosh, S., Williams, M. J., Cuddy, W. S., Simmonds, J., Rey, M. D., et al., (2018). Speed breeding is a powerful tool to accelerate crop research and breeding. Nat. Plants 4 (1), 23. doi: 10.1038/s41477-017-00838

Watson, A., Ghosh, S., Williams, M.J. et al., 2018. Speed breeding is a powerful tool to accelerate crop research and breeding. Nature Plants 4, 23-29 doi:10.1038/s41477-017-0083-8 https://www.biorxiv.org/content/biorxiv /early/2017/07/09/161182.full.pdf

\section{How to cite this article:}

Sai Nayan Raju, Ch. and Kalyan Sagar, C. 2020. Speed Breeding in Agriculture Future Prospects. Int.J.Curr.Microbiol.App.Sci. 9(12): 1059-1076.

doi: https://doi.org/10.20546/ijcmas.2020.912.128 